\title{
Repercussões anatomofisiológicas em recém-nascidos expostos a drogas ilícitas no período gestacional: revisão narrativa
}

\section{Anatomophysiological repercussions in newborns exposed to illicit drugs in the gestational period: narrative review}

Sandra Mary Silva Barbosa ${ }^{1}$. Taiane da Silva Soares ${ }^{2}$. Naesio Ramos de Oliveira ${ }^{3}$. Elisete Mendes Carvalho ${ }^{4}$. Antônia Ionésia Araújo do Amaral ${ }^{5}$. João Joaquim Freitas do Amaral ${ }^{6}$. Maxsuênia Queiroz Medeiros ${ }^{7}$. Francisco Herlânio Costa Carvalho ${ }^{8}$.

1 Fisioterapeuta, Mestranda em Saúde Pública, Universidade Federal do Ceará (UFC), Fortaleza, Ceará, Brasil. 2 Fisioterapeuta, Pós-graduanda em Fisioterapia em Terapia Intensiva, Faculdade Inspirar, Fortaleza, Ceará, Brasil. 3 Graduando em Fisioterapia, Faculdade de Tecnologia Intensiva (FATECI), Fortaleza, Ceará, Brasil. 4 Doutora em Farmacologia, Docente do Curso de Fisioterapia, Universidade Federal do Ceará (UFC). Fisioterapeuta, Maternidade Escola Assis Chateaubriand (MEAC), Fortaleza, Ceará, Brasil. 5 Mestre em Medicina (Ginecologia), Docente da Universidade Federal do Ceará (UFC), Fortaleza, Ceará, Brasil. 6 Doutor em Epidemiologia, Docente Adjunto em Pediatria, Universidade Federal do Ceará (UFC). Médico Pediatra, Hospital Infantil Albert Sabin, Fortaleza, Ceará, Brasil. 7 Mestre em Saúde Pública, Docente do Curso de Fisioterapia, Faculdade de Tecnologia Intensiva (FATECI). Fisioterapeuta, Maternidade Escola Assis Chateaubriand (MEAC), Fortaleza, Ceará, Brasil. 8 Doutor em Medicina (Obstetrícia), Docente do Programa de Pós-graduação em Saúde Coletiva, Universidade Federal do Ceará (UFC). Médico Obstetra, Maternidade Escola Assis Chateaubriand (MEAC), Fortaleza, Ceará, Brasil.

\section{RESUMO}

Introdução: a exposição do feto a drogas ilícitas ainda na gestação pode acarretar em malformações, deformidades e efeitos deletérios ao sistema nervoso central do recém-nascido $(\mathrm{RN})$. Objetivo: realizar uma revisão de literatura acerca das repercussões anatomofisiológicas em recém-nascidos causados pelo uso de drogas ilícitas no período gestacional. Material e Métodos: estudo de revisão narrativa com busca nas bases de dados eletrônicas LILACS, BIREME, MEDLINE, SCIELO e a CAPES, livros, teses, publicações e/ou documentos publicados por órgãos de saúde com período entre 2000 e 2017, nos idiomas português, espanhol e inglês. Foram utilizados os seguintes descritores: recém-nascido, drogas ilícitas, gravidez, anormalidades induzidas por medicamentos e excluídos materiais que trouxessem hipóteses inespecíficas ou as alterações neonatais como extensão adulta descrita pela literatura. Resultados e Discussão: as drogas ilícitas podem atravessar a barreira placentária e hematoencefálica fetal, levando a repercussões importantes no período pós-natal, como a Síndrome de Abstinência Neonatal (SAN), alterações no desenvolvimento da primeira infância, comprometimento neurocomportamental, respiratório e malformações congênitas. Conclusão: um recém-nascido em situação de risco para drogas pode apresentar diversas alterações neurológicas, comportamentais, respiratórias, cardiovasculares, físicas e cinético-funcionais, não podendo afirmar se as alterações neurológicas e comportamentais são permanentes, mas especula-se que haja prejuízos em longo prazo.

Palavras-chave: Recém-nascido. Drogas ilícitas. Gravidez. Anormalidades induzidas por medicamentos.

\section{ABSTRACT}

Introduction: The exposure of the fetus to illicit drugs at the gestational period may lead to malformations, deformities and deleterious effects on the central nervous system of the newborn. Objective: To carry out a review of the literature on the anatomical and physiological repercussions in newborns caused by the use of illicit drugs in the gestational period. Material and Methods: Study of narrative review with researches in the electronic databases LILACS, BIREME, MEDLINE, SCIELO and CAPES, books, theses, publications and/or documents published by health agencies between 2000 and 2017 in Portuguese, Spanish and English language. Were used the descriptors: newborns, illicit drugs, pregnancy, drug-induced abnormalities, and excluded materials that had nonspecific hypotheses or neonatal associations as an adult extension described in the literature. Results and Discussion: Illicit drugs can cross the placental and fetal blood-brain barrier, leading to important postnatal repercussions, such as Neonatal Abstinence Syndrome (SAN), early childhood development, neurobehavioral and respiratory impairment, and congenital malformations. Conclusion: A newborn at risk for drugs may present several neurological, behavioral, respiratory, cardiovascular, physical and kinetics-functional alterations, and it cannot be stated whether the neurological and behavioral changes are permanent, but it is speculated that there are long-term losses.

Keywords: Newborn. Illicit drugs. Pregnancy. Abnormalities induced by medications.

Autor correspondente: Sandra Mary Silva Barbosa, Rua 501, 72, Segunda Etapa, Conjunto Ceará, Fortaleza, Ceará. CEP: 60531-250. Telefone: +55 85 98898-8381. E-mail: sandramary.sb@hotmail.com

Conflito de interesses: Não há qualquer conflito de interesses por parte de qualquer um dos autores.

Recebido em: 06 Nov 2017; Revisado em: 18 Mai 2018; Aceito em: 16 Jun 2018. 


\section{INTRODUÇÃO}

Há muitos anos o uso de drogas vem ultrapassando a linha das margens da sociedade e envolvendo fortemente aspectos políticos e médicos, tornando-se atualmente um dos mais graves problemas de saúde pública a nível mundial. Entre jovens dependentes químicos a overdose é uma das principais causas de morte. É cada vez mais frequente a convivência direta ou indireta com o uso dessas substâncias psicoativas (SPA) e suas consequências. ${ }^{1-3}$

Dados disponibilizados pelo World Drug Report, ${ }^{3}$ demonstram que em 2013 houve estimativa de que 246 milhões de pessoas fizeram uso de drogas ilícitas, correspondendo a pouco mais de $5 \%$ da população mundial, com idade entre 15 e 64 anos. Desses, 27 milhões fazem uso indiscriminado de drogas sendo que os homens estão em situação de vulnerabilidade três vezes maior para uso de maconha, cocaína e anfetaminas, enquanto as mulheres seriam mais propensas ao uso de opioides e tranquilizantes.

O Brasil é o segundo maior consumidor de cocaína, crack e outros derivados (2,8 milhões de pessoas), atrás apenas dos Estados Unidos (4,1 milhões de usuários). Mais de seis milhões de brasileiros já fizeram uso de modo experimental de cocaína ou derivados ao longo da vida. Entre esse grupo, dois milhões fumaram crack, óxi ou merla esporadicamente e um milhão foi usuário de alguma dessas três drogas no último ano. ${ }^{4}$

O II Levantamento Domiciliar sobre o uso de drogas psicotrópicas no Brasil (2005) $)^{4}$ entrevistou 7.939 pessoas com faixa etária de 12 a 65 anos, em 108 cidades brasileiras e estimou que $22,8 \%$ da população total (169.800.000), pode ter feito uso de drogas, excetuando álcool e tabaco, durante sua vida. Dentre os entrevistados, $68,3 \%$ eram do sexo feminino das quais $0,2 \%$ fazia uso de crack e $79,3 \%$ tinha como característica o uso de risco ou nocivo para a saúde. Houve um aumento na prevalência do uso de, pelo menos, 10 substâncias entre as mulheres.

Dentro desse contexto vemos um crescente número de mulheres em idade fértil, em situação de vulnerabilidade para drogas, estando, portanto, expostas a possibilitar que seus conceptos sejam expostos a consequências sociais, culturais, econômicas e, principalmente, biológicas, podendo comprometer seu desenvolvimento físico, psíquico e mental em consequência da exposição precoce a tais substâncias. ${ }^{1}$

Dados epidemiológicos de um relatório brasileiro sobre drogas de 2009 teve como base um levantamento domiciliar realizado no ano de 2005 pela Secretaria Nacional de Políticas sobre Drogas (SENAD). O relatório apresentou um aumento da prevalência de mulheres na faixa etária de 12 a 17 anos com história de consumo de drogas, essas relataram ter consumido bebida alcoólica e outras drogas, pelo menos uma vez na vida. ${ }^{5}$

A população feminina no Brasil possui altos índices do uso de drogas, principalmente álcool e cocaína, sendo consumida na forma de pó ou impura, como o crack e merla. O uso de SPA pelas mulheres está intimamente ligado ao empobrecimento, situação social, ausência de moradia, situação de rua, violência doméstica, transtornos mentais, multiparidade e comorbidades obstétricas. ${ }^{6}$

O uso de drogas pelo sexo feminino, em geral, está associado a situações específicas, como prostituição, violência física e sexual vivenciada na infância ou adolescência, além de problemas crônicos de saúde, físicos ou mentais. Tal fato não é levado em consideração nos serviços de atenção a usuários de drogas, uma vez que o planejamento e a implantação de programas de intervenção para os usuários são baseados nas necessidades masculinas, não sendo analisadas e diferenciadas as necessidades entre os gêneros, sejam fisiológicas, psicológicas ou sociais. ${ }^{7,8}$

$\mathrm{Na}$ mulher gestante, as drogas têm ações diferenciadas no organismo quando comparadas àquelas não grávidas, em decorrência das alterações fisiológicas na metabolização pela presença do feto em crescimento no útero. Assim, o período gravídico em si, é acompanhado por um conjunto de variações emocionais e alterações orgânicas que compõem esse ciclo de forma natural, como as alterações na absorção do sistema gastrointestinal, na dinâmica ventilatória e volume respiratório, alterações hemodinâmicas no transporte e distribuição e queda nos níveis de albumina no primeiro trimestre da gestação. Todo o processo de mudança no organismo grávido modifica a absorção e distribuição de substâncias. ${ }^{9}$

A exposição a substâncias pela gestante se reflete no feto e seus efeitos irão depender do princípio ativo e farmacodinâmico, do período de exposição da gravidez, da frequência e da dose utilizada, podendo resultar em abortamento, morte ou malformação fetal. É importante lembrar que o uso de substâncias ilícitas ou mesmo lícitas como, por exemplo, o cigarro e oálcool não são recomendados, principalmente durante a gravidez. Mesmo medicamentos devem ser administrados com atenção e sob recomendação médica rigorosa, pois ainda não se tem conhecimento claro dos níveis seguros do uso de muitas dessas substâncias no período gestacional. ${ }^{10}$

A vulnerabilidade da gestante ao uso de substâncias psicoativas pode ocasionar complicações perinatais, como a hipoperfusão uterina e placentária, podendo desencadear restrição do desenvolvimento intrauterino, descolamento prematuro da placenta e ruptura prematura de membranas ovulares. O uso dessas substâncias durante a gestação pode desencadear alterações estruturais e fisiológicas fetais, bem como abortamentos, morbimortalidade materna e neonatal. ${ }^{11}$

A maioria das drogas ultrapassa a barreira placentária e hematoencefálica sem haver um processamento prévio, atuando principalmente sobre o sistema nervoso central, causando alterações cognitivas ao recém-nascido, malformações ou síndromes de abstinência neonatal. ${ }^{12}$ A exposição do feto a essas substâncias pode ocasionar malformações e deformidades e outras complicações possíveis como, anemia, hipertensão 
neonatal persistente, hemorragia intraventricular, isquemia miocárdica transitória e arritmias ${ }^{13}$ taquidispneia transitória, apneias e retinopatia. Alterações no desenvolvimento também podem ser percebidas, como: sucção inadequada, hipertonia, tremores, punho cerrado, mioclonias, convulsões e outras alterações neurológicas, além da possibilidade de ocorrência de morte súbita no recém-nascido $(\mathrm{RN}) .^{1,14}$

Os efeitos deletérios causados ao sistema nervoso central dos RN, em decorrência das condições de risco e exposição precoce às drogas por parte das mães na fase intrauterina, não conseguem ser reduzidos depois de instalados, mesmo depois de retirados da situação inicial. Rugolo et al. ${ }^{15}$ explicam que o nascimento provoca uma queda abrupta nos níveis circulantes da droga do compartimento fetal para o recém-nascido, podendo levá-lo à Síndrome de Abstinência. Em casos extremos, podem ser percebidos sinais clínicos acentuados e sintomas da presença da droga e metabólitos na circulação do RN. Tais sinais podem persistir por cerca de três a sete dias.

O uso regular de cocaína pode levar a prematuridade e restrição no crescimento intrauterino, além de uma série de anomalias congênitas como hidrocefalia, microcefalia, problemas cardíacos, fissura palatina, alterações no aparelho digestivo e urinário e deformidades distais, mostrando, assim, que seu uso tem uma ação tóxica direta sobre o desenvolvimento fetal. ${ }^{16}$

Este estudo tem como objetivo realizar uma revisão da literatura acerca das repercussões anatomofisiológicas em recém-nascidos causados pelo uso de drogas ilícitas no período gestacional.

\section{MATERIAL E MÉTODOS}

Trata-se de uma revisão narrativa acerca das repercussões anatomofisiológicas em recém-nascidos expostos a drogas ilícitas no período gestacional. A busca das produções científicas foi realizada nas bases de dados eletrônicas LILACS, BIREME, MEDLINE, SCIELO e a CAPES, livros, teses, publicações e/ou documentos publicados por órgãos de saúde no período entre 2000 e 2017, nos idiomas português, espanhol e inglês. A busca ampliada justifica-se pela escassez de material específico sobre a temática. Foram utilizados os seguintes descritores, de forma isolada ou combinada: "recém-nascido", "drogas ilícitas", "gravidez" e "anormalidades induzidas por medicamentos".

Foi excluído qualquer material que trouxesse hipóteses inespecíficas ou que tratassem as alterações neonatais como uma extensão adulta descrita pela literatura.

\section{RESULTADOS E DISCUSSÃO}

As drogas ilícitas podem atravessar a barreira placentária e hematoencefálica fetal, levando a repercussões importantes no período pós-natal, dentre as quais podem se destacar a Síndrome de Abstinência Neonatal (SAN), alterações no desenvolvimento da primeira infância, comprometimento neurocomportamental e respiratório e malformações congênitas.

\section{Síndrome de Abstinência Neonatal (SAN)}

Uma criança recém-nascida em condições de risco para drogas no período gestacional, sendo retirada dessa situação inicial, não tem reduzido os efeitos deletérios ao sistema nervoso central, uma vez que esses já estejam instalados. A SAN é uma das complicações mais frequentes para o neonato. ${ }^{17} \mathrm{Um}$ estudo realizado em banco de dados na Austrália Ocidental entre os anos de 1980-2005 demonstrou um aumento na prevalência de SAN de 0,97 para 42,2 casos por 10.000 nascidos vivos, tendo seu ápice no ano $2002 .{ }^{18}$

Os sintomas da crise de abstinência podem estar presentes desde o período fetal até o neonatal, manifestando-se desde as primeiras horas, atingindo o pico entre o $3^{\circ}$ e o $14^{\circ}$ dia de vida. ${ }^{19}$ Em casos extremos, podem ser percebidos acentuados sinais clínicos e sintomas da presença da droga e metabólitos na circulação do RN podendo persistir por cerca de três a sete $\operatorname{dias}^{15}$

A apresentação de sinais atípicos compatíveis com abstinência ou privação de drogas pode variar de acordo com o período, intensidade e tipo de droga consumida pela gestante, de modo que o quadro de SAN ocorre com maior frequência em recém-nascidos a termo. ${ }^{19}$

\section{Malformações congênitas - Alterações no crescimento/ desenvolvimento}

Existe consenso a respeito da ocorrência de malformações congênitas causadas pelo consumo de drogas lícitas e ilícitas. Segundo a equipe do Sistema para Detecção de uso Abusivo e Dependência de Substâncias Psicoativas - SUPERA, ${ }^{20}$ o consumo de álcool, tabaco e outras drogas durante o período gestacional pode causar muitos prejuízos para o binômio, mãe-bebê. As malformações congênitas são apontadas como as maiores consequências para o uso de drogas. Para que isso aconteça, especula-se que o consumo deve ocorrer durante o período de proliferação celular.

O álcool é uma das substâncias mais bem estudadas no que se refere aos seus efeitos no período gestacional e nos recém-nascidos, possibilitando que seus prejuízos para o feto estejam bem estabelecidos na literatura. O uso dessa substância pode acarretar o Distúrbio do Espectro da Síndrome Alcoólica Fetal (DESAF). DESAF é uma expressão utilizada para descrever uma diversidade de deficiências e diagnósticos. Dentre os possíveis diagnósticos estão a Síndrome Alcoólica Fetal (SAF) e o Distúrbio de Desenvolvimento Neural Associado ao Álcool (DDNA). ${ }^{20}$

A exposição regular do feto ao álcool tem sido associada à malformação facial, como: depressão vertical acima do lábio superior ausente ou indistinta, lábio superior fino e hipotelorismo ou aproximação dos olhos; além de retardo mental.

Em relação ao uso de tabaco, seus efeitos podem se relacionar com baixo peso ao nascer, redução da circunferência craniana, síndrome da morte súbita infantil, asma, infecções 
respiratórias, redução de Quociente de Inteligência (QI) e distúrbios do comportamento. ${ }^{20}$

A cocaína, o crack e seus derivados podem ocasionar problemas no processo gestacional e no desenvolvimento do feto. O uso regular pode levar a prematuridade e restrição no crescimento intrauterino além de uma série de anomalias congênitas como hidrocefalia, microcefalia, problemas cardíacos, fissura palatina, alterações no aparelho digestivo e urinário e deformidades distais. Mostrando assim a ação tóxica direta que a cocaína tem sobre o desenvolvimento fetal. ${ }^{16}$

Outras complicações possíveis são anemia, hipertensão neonatal persistente, hemorragia intraventricular, isquemia miocárdica transitória e arritmias com alterações eletrocardiográficas. ${ }^{13}$

\section{Comprometimento respiratório}

Em relação aos comprometimentos respiratórios podemos destacar taquidispneia transitória, apneias, desconforto respiratório e edema agudo de pulmão. ${ }^{2}$

\section{Comprometimento neurocomportamental}

No feto, as drogas podem afetar a formação e o remodelamento do cérebro através do efeito inibitório dos neurotransmissores, noradrenalina, dopamina e serotonina nos terminais pré-sinápticos, onde vai haver acúmulo dessas substâncias fazendo com que persistam por tempo maior junto aos receptores dos órgãos efetores, levando a respostas exageradas. Posteriormente, poderá haver depleção deles, principalmente da dopamina. ${ }^{21}$ Esta cascata de acontecimentos pode levar a alterações no crescimento cerebral e na arquitetura do córtex, principalmente com a imprecisão da laminação cortical, sugerindo desordens na diferenciação e migração neuronal e no desenvolvimento do prosencéfalo. ${ }^{13,16}$

Os RNs podem apresentar perturbações neurológicas observadas através da postura, reflexos e movimentação. Estudos mostram que crianças podem apresentar sucção inadequada gerando dificuldades em alimentar-se, hipertonia ou hipotonia, tremores, punho cerrado, mioclonias, sudorese, convulsões, reflexos hiper-reativos e outras alterações neurológicas. ${ }^{13,22}$

Quanto ao comportamento, podem ocorrer nos primeiros dias de vida: choro intenso e estridente de difícil consolação, alterações do sono, irritação alternando com longos períodos de sono profundo, sinais cutâneos, visuais, autonômicos e fisiológicos relativos ao estresse e abstinência..$^{1,14,22}$ Tais sinais podem apontar sutis morbidades neurológicas. ${ }^{16}$

Perturbações no sistema nervoso central podem refletir diretamente sobre outros sistemas, por exemplo: musculoesquelético, gastrointestinal, cardiovascular, respiratório ${ }^{19}$ e sistema regulador (subsistemas). Desse modo, podem surgir sintomas como vômitos, diarreia, desidratação e febre. Tais sintomas são inespecíficos e podem estar presentes em outros problemas médicos.

Não se sabe, até o momento, quais os mecanismos causadores desses efeitos, mas supõe-se que a hipoxemia, as alterações na síntese do Ácido Desoxirribonucleico (DNA) em algumas regiões cerebrais e as alterações nos neurotransmissores possam estar envolvidas. ${ }^{13,23}$

Não pode ser negligenciado o fato de que as alterações encontradas em recém-nascidos em situação de risco para exposição pré-natal a drogas podem ser causadas pela droga em si ou pela negligência nutricional, violência ou doenças sexualmente transmissíveis que geralmente estão presentes neste contexto.

Outras complicações comumente encontradas na literatura são retinopatia, icterícia e sofrimento fetal podendo chegar a morte súbita, em casos mais graves. ${ }^{1,2,14}$

\section{Desenvolvimento da primeira Infância}

Qualquer situação que atrapalhe o andamento normal da evolução de uma gestação pode acarretar consequências graves sobre o RN. Essas consequências, a depender da gravidade, podem perdurar pela infância, adolescência e até a idade adulta. ${ }^{24,25}$

Segundo Pechanscky et al., ${ }^{26}$ crianças expostas a drogas durante o período gestacional podem apresentar patologias ligadas, direta ou indiretamente, à situação dos pais e mais especificamente da mãe quando grávida. As crianças podem evoluir com doenças cardíacas, desordens na pressão arterial (PA), distúrbios metabólicos, desnutrição, distúrbios e malformações no aparelho digestivo e geniturinário. Malformações na face, dedos e membros podem estar presentes e dificultar o desenvolvimento e convívio sócioemocional destas crianças.

Além de trazer prejuízos visíveis ao desenvolvimento, as drogas podem acarretar dificuldades escolares na primeira infância prejudicando seu processo de aprendizado. Esses são de difícil avaliação e qualificação o que os torna ainda mais prejudiciais, pela demora na procura de ajuda especializada. ${ }^{16}$

Estudos realizados pela Brown University School of Medicine, que acompanharam o desenvolvimento de crianças expostas a drogas durante a gestação, evidenciaram a presença de alterações em longo prazo como baixo QI, dificuldades cognitivas, baixo nível de atenção, dificuldades escolares, emocionais e interativas, bem como dificuldades neuromotoras. Autores como Silva, ${ }^{27}$ Amiel-Tison \& Gosselin ${ }^{28}$ e Brazelton ${ }^{29}$ destacam o acompanhamento do desenvolvimento de crianças, com riscos para problemas neurocomportamentais, com o intuito de minimizá-los.

\section{CONCLUSÃO}

Segundo o que se encontrou na literatura, um recém-nascido em situação de risco para drogas pode apresentar uma série 
de alterações neurológicas, comportamentais, respiratórias, cardiovasculares, físicas e cinético-funcionais. Dentre as quais se podem destacar a SAN sendo a mais citada entre os autores.

Mesmo sendo a SAN uma condição passageira, sua presença tem potencial de deixar sequelas e envolver

\section{REFERÊNCIAS}

1. Brasil. Ministério da Justiça. OBID - Observatório Brasileiro de Informações Sobre Drogas [Internet]. Brasília: Ministério da Justiça; 2012 [Acesso em: 19 Out 2016]. Disponível em: http://obid.senad. gov.br/obid

2. Portela GL, Barros LM, Frota NM, Landim AP, Caetano JA, Farias FL. Percepção da gestante sobre o consumo de drogas ilícitas na gestação. Rev Eletrônica Saúde Mental Álcool Drog. 2013;9(2):58-63.

3. United Nations Office on Drugs end Crime. World Drug Report, 2015 [Internet]. Vienna: United Nations publication; 2015 [Acesso em: 19 Out 2016]. Disponível em: https://www.unodc.org/ documents/wdr2015/World_Drug_Report_2015.pdf

4. Universidade Federal de São Paulo (UNIFESP); Instituto Nacional de Ciência e Tecnologia para Políticas Públicas de Álcool e Outras Drogas (INPAD). LENAD - II Levantamento Nacional de Álcool e outras Drogas [Internet]. São Paulo: UNIFESP; 2012 [Acesso em: 22 Out 2016]. Disponível em: http://inpad.org.br/wp-content/ uploads/2014/03/Lenad-II-Relat\%C3\%B3rio.pdf

5. Brasil. Secretaria Nacional de Políticas sobre Drogas (SENAD). Relatório brasileiro sobre drogas [Internet]. Brasília: SENAD; 2009 [Acesso em: 22 Out 2016]. Disponível em: http://obid.senad.gov.br/ obid/biblioteca/publicacoes/relatorio-brasileiro-sobre-drogas-2010. pdf

6. Marangoni SR., Oliveira ML. Uso de crack por multípara em vulnerabilidade social: história de vida. Cienc. Cuid. Saude. 2012;11(1):166-72.

7. Oliveira JF, Paiva MS, Valente CL. Representações sociais de profissionais de saúde sobre o consumo de drogas: um olhar numa perspectiva de gênero. Cien Saude Colet. 2006;11(2):473-81.

8. Prado MA, Queiroz IS. A emergência da politização da intimidade na experiência de mulheres usuárias de drogas. Estudos de Psicologia. 2012;17(2):305-12.

9. Neme B. Obstetrícia Básica. 3. ed. São Paulo: Sarvier; 2006.

10. Rocha RS, Bezerra SC, Lima JW, Costa FS. Consumo de medicamentos, álcool e fumo na gestação e avaliação dos riscos teratogênicos. Rev Gaucha Enferm. 2013;34(2):37-45.

11. Lopes TD, Arruda PP. As repercussões do uso abusivo de drogas no período Gravídico/puerperal. Revista Saúde e Pesquisa. 2010;3(1): 79-83.

12. Kassada DS, Marcon SS, Pagliarini MA. Prevalência do uso de drogas de abuso por gestantes. Acta Paul Enferm. 2013;26(5):467-71.

13. Cunha GB. Exposição pré-natal a cocaína e efeitos neurocomportamentais no recém-nascido [Tese]. Porto Alegre: Universidade Federal do Rio Grande do Sul; 2007. 242 p. todos os sistemas do indivíduo. Sua presença é facilmente perceptível por meio das alterações de comportamento e postura do RN.

Ainda não é possível afirmar que as alterações neurológicas e comportamentais são permanentes, mas especula-se que haja prejuízos em longo prazo.

14. Kopelman BI, Santos AM, Goulart AL, Almeida AF, Miyoshi MH, Guinsburg R. Diagnóstico e tratamento em neonatologia. São Paulo: Editora Atheneu; 2005.

15. Neufel HG. Síndrome de abstinência neonatal. In: Rugolo LM. Manual de neonatologia. São Paulo: Revinter; 2000. p. 69-71.

16. Alencar JC, Alencar CA Junior, Matos AM. "Crack Babies": uma revisão sistemática dos efeitos em recém-nascidos e em crianças do uso do crack durante a gestação. Revista de Pediatria SOPERJ. 2011;12(1):16-21.

17. Padilla JA, Garrido AA, Velázquez FR, Gutiérrez MT, Huizar LM, Hernándes HG, et al. Hijos de madres adictas con síndrome de abstinencia en terapia intensiva neonatal. Bol Med Hosp Infant Mex. 2008;65(4):276-81.

18. Wang M, Rozenkrantz MD. Perinatal Drug Abuse and Neonatal Drug Withdrawal [Internet]. New York: Medscape; 2014 [acesso em: 2016 Set 29]. Disponível em: http://emedicine.medscape.com/ article/978492-overview

19. Ferreira P, Fernandes N. Síndrome de privação neonatal. Revisão da abordagem. Revista Toxicodependências. Edição IDT. 2008;14(1):24-9.

20. Formigoni ML. Efeitos de substâncias psicoativas. 7. ed. Brasília. Secretaria Nacional de Políticas sobre Drogas; 2014.

21. Corradine HB. Cocaína: efeitos nas gestantes e nas crianças. Pediatria. 1996;18(2):170-4.

22. Renner FW, Garcia EL, Renner JD, Costa BP, Figueira FP, Ebert JP, et al. Perfil epidemiológico das puérperas e dos recém-nascidos atendidos na maternidade de um hospital de referência do interior do Rio Grande do Sul no primeiro semestre de 2014. Boletim Científico de Pediatria. 2015;4(2):27-32.

23. Giust J, Mitsuhiru SS, Zelberman ML. Cocaína e gestação. São Paulo: Núcleo Einstein de Álcool e Drogas do Hospital Israelita Albert Einstein; 2012.

24. Brasil. Ministério da Saúde. Pré-natal e puerpério: atenção qualificada e humanizada: manual técnico [Internet]. Brasília: Ministério da Saúde; 2005 [Acesso em: 22 Out 2016]. Disponível em: http://bvsms.saude.gov.br/bvs/publicacoes/manual_pre_natal_ puerperio_3ed.pdf

25. Brasil. Ministério da Saúde. Atenção humanizada ao recém-nascido de baixo peso - método canguru: manual técnico [Internet]. Brasília: Ministério da Saúde; 2013 [Acesso em: 22 Out 2016]. Disponível em: http://bvsms.saude.gov.br/bvs/publicacoes/ atencao_humanizada_recem_nascido_canguru.pdf

26. Pechanscky F, Diemen LV, Micheli D, Amaral MB. O uso de 
substâncias psicoativas no Brasil: módulo 1. 7. Ed. Brasília: Secretaria Nacional de Políticas sobre Drogas; 2014. Capítulo 4, Fatores de risco em diferentes grupos de usuários: mulheres, adolescentes, idosos e indígenas; p. 67.

27. Dubowitz LM, Dubowitz V, Mercuri E. The neurological assessmentof the preterm \& full term newborn infant. London: Ed. Cambridge; 1999.
28. Amiel-Tison C, Gosselin J; Louércio SD, tradutora Avaliação neurológica do nascimento aos 6 anos. 2 ed. Porto Alegre: Artmed; 2009.

29. Brazelton TB, Nugent K. The neonatal behavioral assessment scale. Boston: Mac Keith Press; 2011.

\section{Como citar:}

Barbosa SM, Soares TS, Oliveira NR, Carvalho EM, Amaral AI, Amaral JJ, et al. Repercussões anatomofisiológicas em recém-nascidos expostos a drogas ilícitas no período gestacional: revisão narrativa. Rev Med UFC. 2018 out-dez;58(4):46-51. 